\title{
Identification of Rice Quality Through Pattern Classification Using Computer Vision Image Processing
}

\author{
Muhammad Tariq Muhammad Saleem Akhtar Muhammad Saleem Anjum \\ Department of Computer Science, Minhaj University Lahore, Punjab, Pakistan
}

\begin{abstract}
Rice is the source of Pakistan agriculture industry and food. For agriculture, industry and the oldest sector in the world use rice for different purpose. There are many challenges in the particular sector such as their analysis. This analysis mostly often related to its texture, color, shape, grain etc. In this study, Vision system used to check the quality of rice using some texture features such as color, shape and characteristics. In this study Computer Vision Image Processing tool applied on three different types of rice. Using this tool we apply pattern classification using nearest neighbor and K-nearest neighbor algorithm. Using these algorithms we get results of three varieties of rice Bastmati, Jasmine and White rice. In both algorithms white rice result show best from Basmati rice and Jasmine rice. White rice result is $93.75 \%$ which is best as quality wise. Other tool also available like as MATLAB, Mazda etc for future more best prediction.
\end{abstract}

Keywords: RST-Invariant features, Histogram features, Texture features, Nearest Neighbor algorithm, Knearest neighbor algorithm

DOI: $10.7176 / \mathrm{CEIS} / 11-2-01$

Publication date: February $29^{\text {th }} 2020$

\section{Introduction}

Rice grows in over one hundred countries with an annual harvest of 158 million hectares, producing more than 700 million tons a year (470 million tons of rice). About 640 million tons of rice is grown in Asia, representing $90 \%$ of the world's production. South Africa weighs about 19 million tons, and Latin America weighs about 25 million tons. In Asia and Sub-Saharan Africa, almost all rice grows on small farms of 0.5-3 ha. The highest yields were usually obtained by planting at high altitudes and during the daytime, where intensive treatment was used, or in lowland areas with high intensity. Southwest Australia, Hokkaido in Japan, Spain, Italy, northern California and the Pacific Delta are the best examples. World sugar production was more than tripled between 1961 and 2010, with a growth rate of $2.24 \%$ per annum $(2.21 \%$ in production Asian studies). The biggest increase in sugar production was due to higher yields, which was about $1.74 \%$ per year compared to the growth rate of $0.49 \%$ for the harvested areas. Specifically, rice production increased by $51.1 \mathrm{~kg} / \mathrm{ha}$ per year, although this growth rate has been reduced in percentage and overall efficiency [1].

More than $90 \%$ of the rice is produced and used in Asia. In terms of consumption of food, what sets Asia apart from the rest of the world is its reliance on rice: a nutritious food for many people, including 560 million people poor in the region. Other regions are more dependent on other foods.

Pakistan is the 11 th largest producer of rice in the world. Pakistan exports about $8 \%$ of the total rice exports to the world. This is a milestone in Pakistan's agriculture. Rice is an important crop of Kharif. In 2016-17, Pakistan received 6.7 million tons of which 4 million were exported to neighboring countries, the Middle East and Africa. Rice grows in the fertile fields of Sindh and Punjab, where millions of farmers rely on rice cultivation as their main source of labor. The most popular varieties grown in Pakistan include basmati, known for their taste and quality. Pakistan is the main producer of this brand [2].

Rice quality testing is one of important role in machine learning. Some researchers suggesting that the face is something more informative than its the appearance of the colors changes dramatically between some objects rather than the form. It is it is impossible to provide a precise measurement. Rice integrity means touch seed when taking samples. Main purpose should be offer another avenue for excellence monitor and analyze the quality of rice, which reducing the effort necessary, cost and time. Rice high value in production farms and plants for identification It is very important that the rice is good. Image action that is important and progressive technology makes things important made in tradition, farmer knows rice flour in detail by naked eyes. They also have learning experience variety [3].

Rice is great importance to the health of the people in our country, as well as to the world in terms of its importance and usefulness. It is also commonly used in business. There are special opportunities for rice production in our country. This is a physical body, a natural feature of blood; aroma, taste and aroma problems as good as real estate. From the point of view of the user, this is the first look that comes to mind from the stand out to the types of rice sold on the market. After production, it recognizes the need for technological methods, as stabilization of rice, stabilization of its species and separation of qualitative elements is not ideal and timeconsuming, especially from the perspective of those in to the extent of the production capacity. Therefore, when we look at recent studies of cereal foods using optical devices and optical processing methods, it can be seen that 
products will be tested on many physical properties, such as the color, style, quality and size. Comparison taken through processed rice by subtraction of area, length and shape of freezer using image analysis [4].

The quality and quantity of rice seed is usually determined based on its various characteristics and characteristics. Classification of rice varieties in a specific area is of utmost importance to the specialists group for assessing the level of morality, as well as improving the quality of rice outside of the country [5].

In order to determine the diversity of the species, different samples are required by inspection of agricultural land by skilled laborers. During the cultivation, harvesting and production, varieties of rice are mixed and mixed, which reduces the authenticity and quality of the products. However, having a large number of different types makes it difficult to test and classify as a new employee. Variations can be distinguished in the field, determining their height, the location of the decay, the type of seed and the color, and so on [6].

The neares neighbor and K-neares neighbor is the best algorithm for classifying seeds. Using these algorithms goal achieved the best results. These methods are easy to understand and provide quick results. In this way find that in the neighboring regions $85 \%$ and $76 \%$ and the neighboring $90 \%$ and $73 \%$ serve as the end of the classification system. The best percentages for classification and provide greater accuracy. In these results, we conclude that $90 \%$ and $85 \%$ are the highest percentage results achieved. Finally, we believe that the closest neighbor and the closest neighbor are the best and fastest method [7].

The object of this research to analysis of different rice varieties through their images and identify best quality of rice. Latest varieties and their growth rate used by different environmental factor like not more raining season sun light or lake or water. So, human perception base perceptions or difficult task to identify these best rice quality varieties. It provides efficient result by using machine learning approach.

\section{LITRATURE REVIEW}

In this study, a qualitative analysis of milled rice using a monitoring device is integrated with several additional genetic models. Images of three different types of rice, including g Low-processed sound grains (LPS), Lowprocessed broken grains (LPB), High-processed sound grains (HPS), and High-processed broken grains (HPB), represent different types of products, there were sales using a computer display system Three different methods, including integrated circuits, heat sensors, test boards, and Bayesian networks, were used to analyze rice samples. Correlation of the results revealed that a neural network with a topology of 12-5*4 yielded the highest classification $(98.72 \%)$. In addition, the heat transfer mechanism and function of the Universal Pearson VII kernel (98.48\%), the end of the REP algorithm (97.50\%) and the Bayesian correlation with the Hill Climber search algorithm $(96.89 \%)$ ) is much higher, respectively. The results presented in this article can be used to develop an efficient system for the complete automation and classification of rice grains [8].

Rice is one of the most consumed foods in the world accounting for more than 60 percent world population. Many rice varieties are grown, imported and exported around the world. Rice different types can be mixed during their production and testing. Many studies have been conducted to classify rice. Types and characteristics of various rice used using images [9].

Species identification problem using destructive devices is much more difficult due to knowledge, position and location. Real study was created to identify eight different types of rice through their own combination and use machine varieties to develop a grape variety verification system. The study was conducted in collaboration with a number of technical experts. Different methods used such as App List, K-Neighbor Neighbor's and NaïveBayes. KNN implemented to other methods with accuracy, accuracy and recall of $99.16 \%, 99.12 \%$ and $99.12 \%$ respectively [6].

It describes an effective method for determining the size of the Oryza sativa L by using sensors along with detection of clean and disconnected cells with high accuracy compared to visitors. A new non-invasive method for detecting high levels of basmati rice seeds using image analysis. Since the error in calculating the percent percentage of regular seeds and the percentage of pearl seeds was $10.59 \%$ and $2.27 \%$, respectively. Therefore, it can be emphasized that the proposed system is $10.59 \%$ and $2.27 \%$ more accurate than the analyst for the evaluation of normal and penile grains. Thus, the proposed approach provides the most successful results compared to the visitor experience. To further improve the quality analysis, additional parameters can be added to increase accuracy [10].

Rice is one of the most widely produced and widely used seeds in the world, which is one of the main sources of food in our country because of its rich nutritional value. In this study, computer software was designed to detect various types of rice. A total of 3810 images of corn rice were obtained for two species, each of which was produced and produced. A total of 7 species were found for each kidney. Using these functions, images were created using machine learning and measurement methods LR, MLP, SVM, DT, RF, NB, and k-NN. Classification success indicators were obtained 93.02\% (LR), 92.86\% (MLP), 92.83\% (SVM), 92.49\% (DT), $92.39 \%(\mathrm{RF}), 91.71 \%(\mathrm{NB}), 88.58 \%(\mathrm{k}-\mathrm{NN})$. When we look at the results of a successful acquisition, we can say that the study was successful [11].

Advanced programming techniques such as image processing, image enhancement, optimization, and 
optimization are used to analyze the type of rice. The next example is the use of templates for textbooks and graphics. Finally, a mass support heat transfer machine (M-SVM) was developed to identify the type of rice based on the correlation between the main characteristics of the training and image research. These rice classifications are as accurate and complex as all other classification systems [12].

Productivity plays an important role in the global economy. Information technology is one of the tools to achieve this. With the tools available, we provide computer vision solutions, along with sophisticated intelligent algorithms that have achieved significant results in finding patterns in images. To this end, this work contains a physical analysis, whose purpose is to determine the usefulness of computer vision in the field of agriculture for the production of the five most productive worlds: corn, rice, wheat, soy and barley. To this end, we present 25 articles selected over the past five years, with various approaches to the treatment of areas related to disease monitoring, cardiac quality and specialty. Based on the result of an annual review, one can identify the best opportunities, such as using a prototype (GPU) and more sophisticated methods, such as DBN (Deep Belief Networks), to create computer-aided communication methods. It is used for stabilizing the farm [13].

Algorithms were developed to remove it thirteen looks, six colors and fifteen of color images of heart images. This is another convenient interface designed for each platform and for providing custom templates. High the accuracy of the classification was given by telephone in morphological and color form features. A total classification of $92 \%$ was obtained from the combination eg. Exact face classification AT307, BG250, BG358, BG450, BW262, BW267, W361, BW363 and BW364 accounted for 94\%, 98\%, 84\%, 100\%, 94\%, 68\%, 98\%, $94 \%$ and $94 \%$ respectively. Internal differences were noted artists often come up with the right ideas [4].

We propose and empirically evaluate Rice-Paddy quality to address the various shortcomings of traditional quality analysis methods. We compare their quality during the design process with the measurement. No copies of the rice field were taken anywhere from a bag placed in front of the camera to determine the quality of rice for price analysis. An accurate and efficient material processing system has been developed that provides methods comparable to the current standard. Encoding protocols were established and the review was consistent. This can be used in real-time applications where the object must look for prefixes in its pipeline. An important feature is the study of software in a series of videos for use in testing applications. Adding a constant connection to the body provides a smoother and more efficient release than a single signal [14].

Early detection and accurate diagnosis of antibiotics is an important step in the process of crop protection. To address these problems, the article provides the use of machine learning models to control and classify diseases. In this article, we focus on the cultivation of rice (Oryza sativa). Examples of pathogens in leaves and fats were found in rice. We have collected 619 varieties of sugar from the field in three classes : (a) Rice Blast (RB), (b) Bacterial Leaf Blight (BLB), (c) Sheat Blight (SB) and (d) Healthy Leave (HL). We have used a pretrained deep convolutional neural network $(\mathrm{CNN})$ as a reference and a vector machine $(\mathrm{SVM})$ as our target. We found some encouraging results. Early detection of viruses in this way can be used as a precautionary measure as a precautionary measure. In addition, rice production can be increased by establishing special treatment on crop yields [15].

Seed classification is the process by which different types of seeds are classified into different classes based on their characteristics. In this paper, we analyzed seeds using the Weka tool. Data was obtained from the UCI website. Features of the core used: area, perimeter, compactness, core length, core width, asymmetry coefficient and wavelength. In the Weka classification, we use the Just, Bayes, Meta, and Lazy methods. The classifiers used in these methods are multilayer perceptron, logistics, SMOs, updated Naïve Bayes, Bayesian flexible networks, Bayesian networks, multiclass classifier, regression and LWL classification. After that, we used 10 sheets, 5 sheets and 2 color checks, as well as the installation method. The multilayer Perceptron and Logistics from the operating system provide higher performance than all other classes, which is $95.2 \%$ for all users of the $10 \mathrm{x}$ validation cross. We also evaluate the result using 5-fold and 2-fold cross-acceptance, but we note that overall productivity decreases with decreasing herd values, with the exception of the multilayer Perceptron classifier given a critical accuracy level of 97.6. \% not used 5 Cross Cross Recognition. The multilayer perceptron also provides the highest possible value compared to using the US state, which is $99.5 \%$, and printing provides the second largest overall accuracy of $98.6 \%$. Finally, we also note that state development provides a higher level of productivity than the main development in the classification process [16].

Rice is one of the largest and most important crops for people in Asian countries. In the international market and in countries, the mode of transport is evaluated using quality rice. Therefore, it is more important to indicate quality. Confirmation of the quality of cheese made by the hands of visitors guarantees some degree of accuracy. But this requires a lot of human strength, it is important to take a lot of time and judge. The rice model is a mixture of rice, barley rice, rice husk, rice fields, stone and foreign ingredients. A rice dish should be divided into these six categories to determine the quality of rice. This article presents a method for isolating and classifying rice samples from rice based on existing colors and characteristics using image processing and training methods. This method begins by acquiring the features of a CCD camera. The distribution and classification ratio was $96.0 \%$ and $88.0 \%$, respectively [17] 
The challenge of determining the best seeds to grow is the variety of canola seeds. In this article, we strive to present different teaching methods for analyzing different types of canola seeds, which enable people or farmers to identify different types of canola. Canola kernels are used by image processing computer equipment. We have 4 different varieties of canola seeds Gobi Sarson (A), Barassica comp (B), Sathri (C) and Rocket Herbof (D), and we photograph canola seeds from these different varieties. Each species has 10 statues, and each contains $10 * 4=40$ canola seeds. we board the train and look for information on all varieties of canola seeds. He then models and analyzes the data sets that will be compared for the classification model, and uses the algorithms of neighboring boundaries and final boundaries for the final separation in computer processing tools. We find that in the neighboring regions $85 \%$ and $76 \%$ and the neighboring $90 \%$ and $73 \%$ serve as the end of the classification system. The best percentages for classification and provide greater accuracy. It is important for the farmer and other people to identify different canola seeds [7].

We take ten different photographs of each type of tree. The capacity and information of visitors is critical to completing this process. The whole process will go through the production of computer products. In this tool we use the "History", "Package" and "Arithmetic" settings. We obtained final results for different types of plants used near the $82 \%$ border and at the border close to $\mathrm{k}=2$, and reached a load of $82 \%$ when $=\mathrm{k}=3$, the ratio $82 \%$ where $\mathrm{k}=4$ denotes the formula $76 \%$, and where $\mathrm{k}=5$, an average percentage of $72 \%$ of the result gives the highest accuracy and classifies plants. Interest is optimized using these classification algorithms. In this way, we can easily classify different plants, and these methods also enable the farmer and other people to identify and select the different plants that are best suited for the best results [18].

Nearest Neighbor and K-near neighbor are the best algorithm for classifying canola seeds and it give the best results. These methods are easy to understand and provide quick results. We find that in the neighboring regions $85 \%$ and $76 \%$ and the neighboring $90 \%$ and $73 \%$ serve as the end of the classification system. The best percentages for classification and provide greater accuracy. In these results, we find that $90 \%$ and $85 \%$ of the findings are the highest percentage results found in our study. Finally, we believe that the closest neighbor and the closest neighbor are the best and fastest method [19].

A large number of studies have been conducted to classify plant species and to identify diseases of various crops using specific images. The physical problem of identity verification is further complicated by the general problems of object recognition due to its understanding, placement, and organization. This study was conducted to identify three different types of rice, using different groups to collect and use the methods used. There are various methods that can vary from simple to complex. However, the study was conducted on specific models, such as discriminant analysis, logistic disturbances, K-Neighbors and Naive-Bayes neighbors. The index was made using other methods with an accuracy and accuracy of $99.16 \%$ and $99 \%$, respectively [20].

\section{METHDOLOGY}

In this study, data will be collected physically and we use the quantitative research. Different images of rice are taken by digital camera. The three varieties of rice seeds will be used in this research. We get nine different images of each kind of rice. In this way we use the $9 * 3=27$ images of rice seeds. In a figure the one snapshot of each kind of rice variety shown:

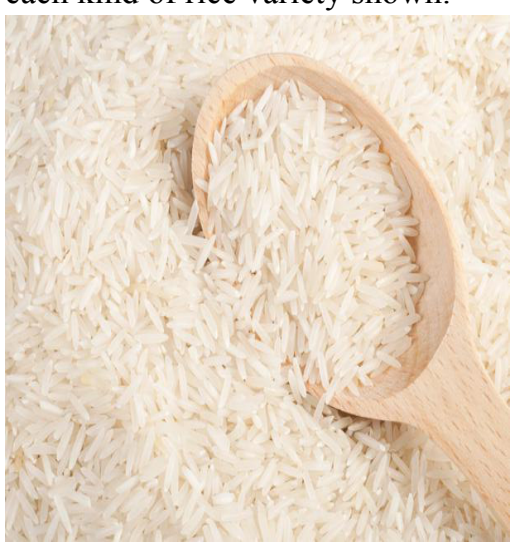

Basmati Rice

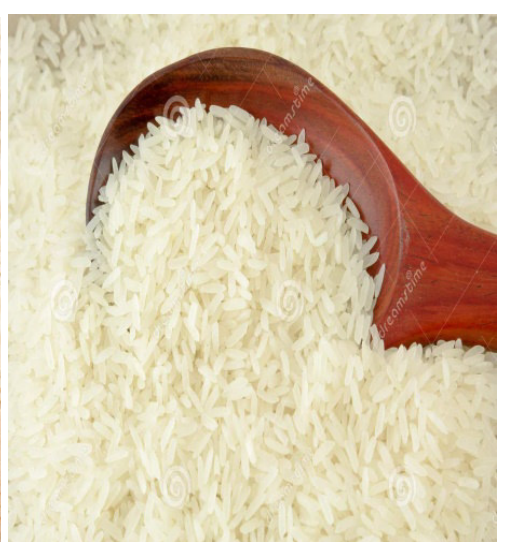

Jasmine Rice

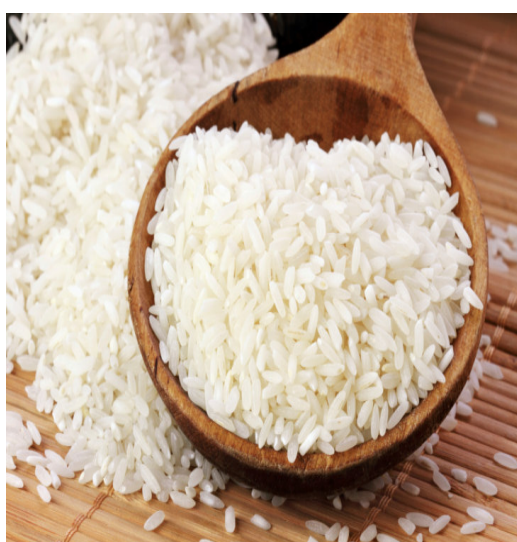

White Rice

In first step image preprocessing will be done. In next step image is analyzed by using texture base analysis. Then at the end by applying different machine learning algorithm like as nearest neighbor algorithm and Knearest neighbor algorithm .For using these approaches we process every image and get results in following way: 


\section{Machine Learning}

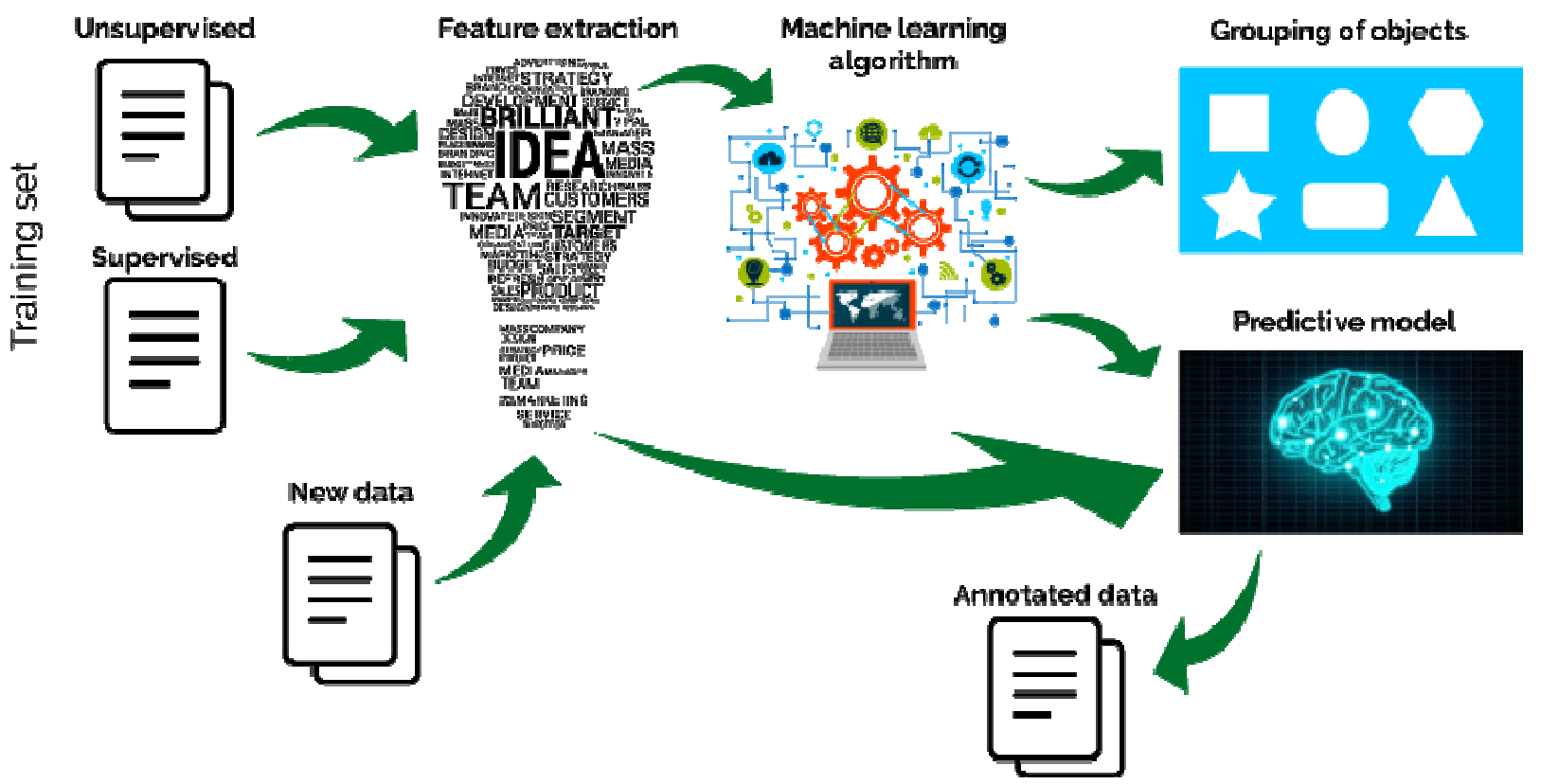

In a supervised learning, we are assumed a set of example pairs and the object is to find a function in the allowable class of functions those both the examples.

Using unsupervised learning, some data $x$ is assumed or given and the cost function can be minimized, that will be any function of the data $\boldsymbol{x}$ and the network's output.

\section{CVIP Tool}

In this study we use CVIP tool 5.7e version which is GUI-based software where the user can explore image analysis, enhancement, restoration and compression algorithms. CVIP tools supports the standard image processing functions such as image restoration, image compression, logical and arithmetical operations between images, image sharpening, Frequency transform, edge detection, contrast manipulation, segmentation and geometric transformations. Using this version of computer vision image processing tool we step by step precede our work which snapshots attached below and explained.

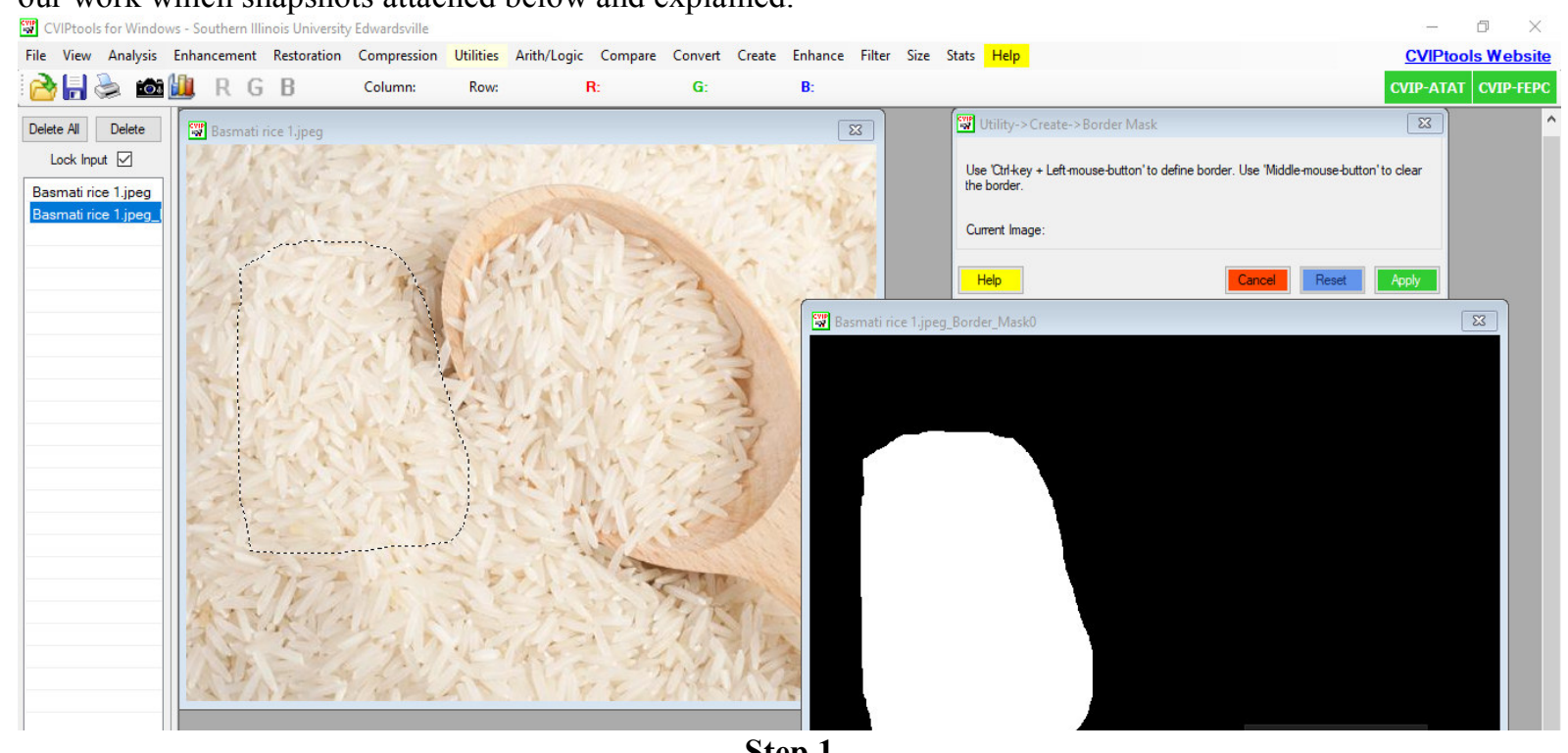

In first step of CVIP tool we open image in CVIP tool and create different border masks of image for apply different features results. In CVIP tool border mask option is defined in utilities. 
:

File View Analysis Enhancement Restoration Compression Utilties Arith/Logic Compare Convert Create Enhance Filter Size Stats Help

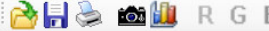

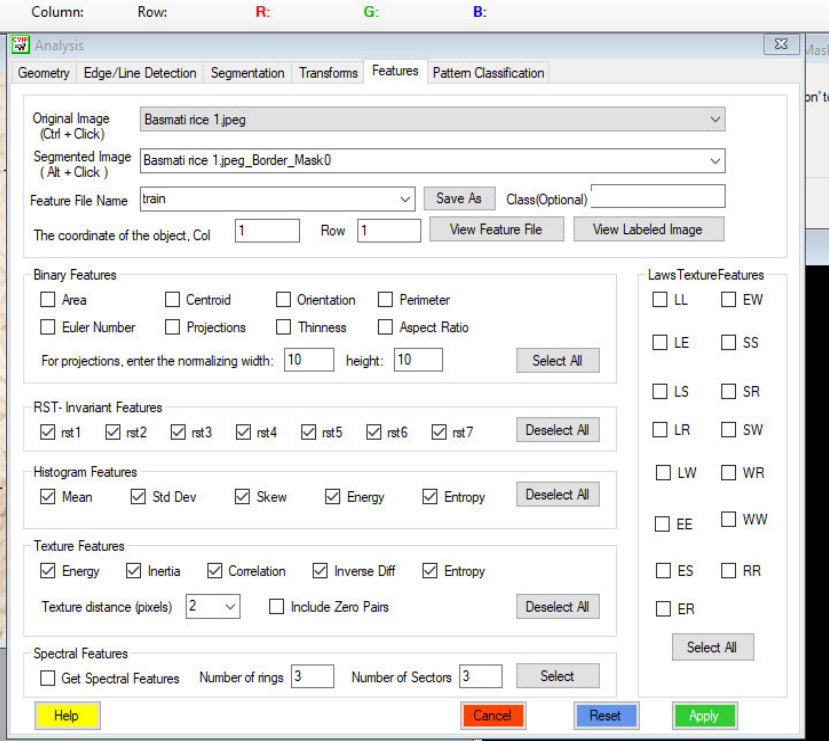

\section{Step 2}

In step 2 of CVIP tool after taking the different border masks of images we apply RST-Invariant feature, Histogram features and Texture features. In this step we get sub feature results of selected images border masks. 梀

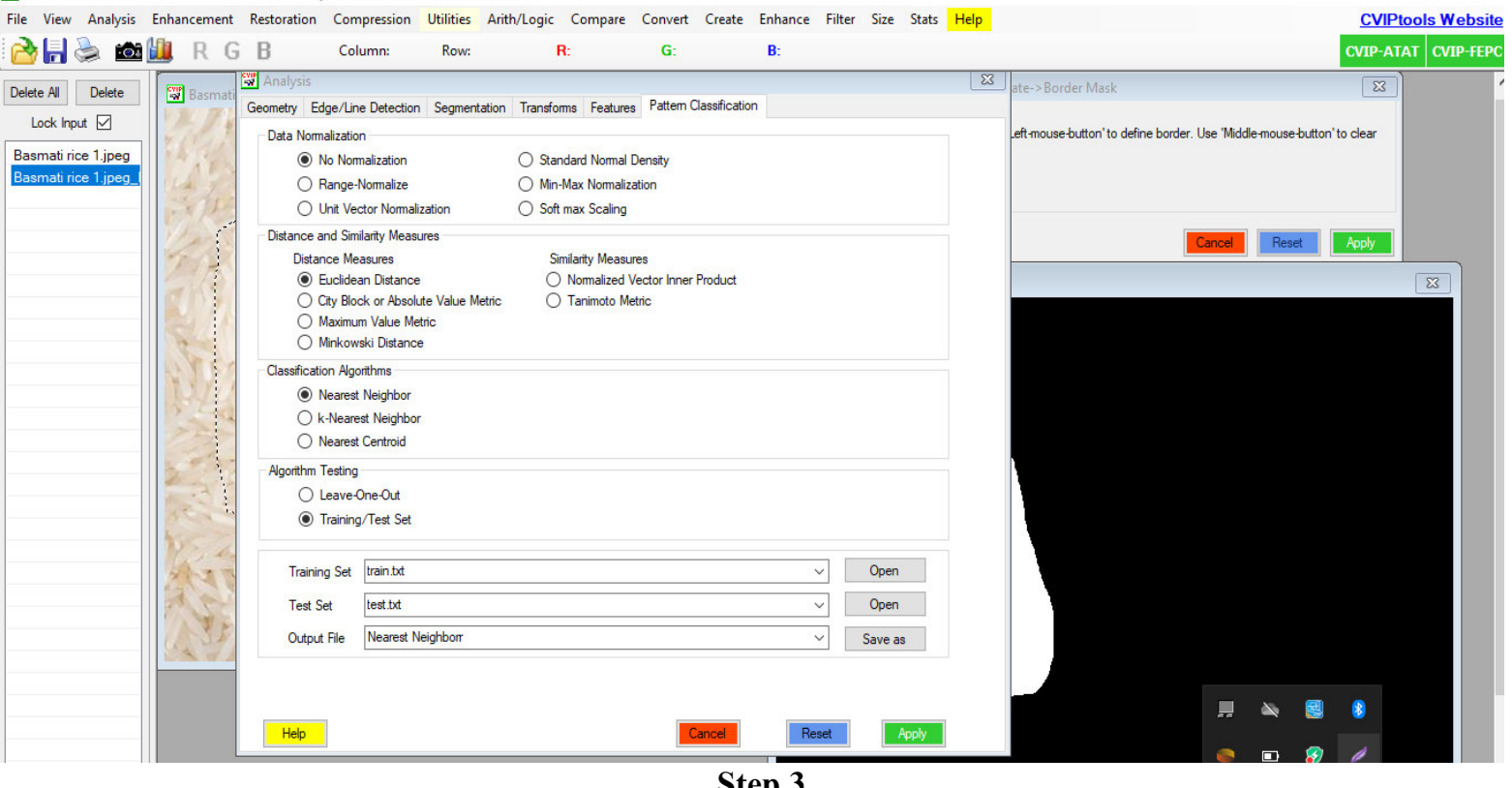

Step 3

After getting the features results we compare train data set and test data set through apply the nearest neighbor algorithm and K-nearest neighbor algorithm. In this step final results will be achieved of different rice varieties. 


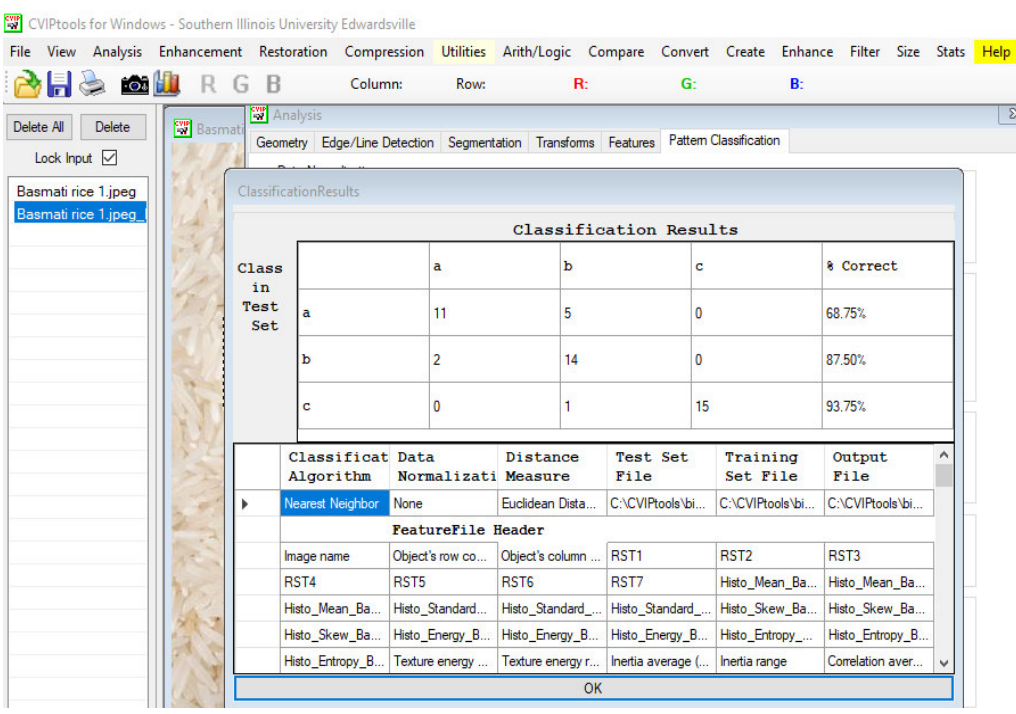

\section{Step 4}

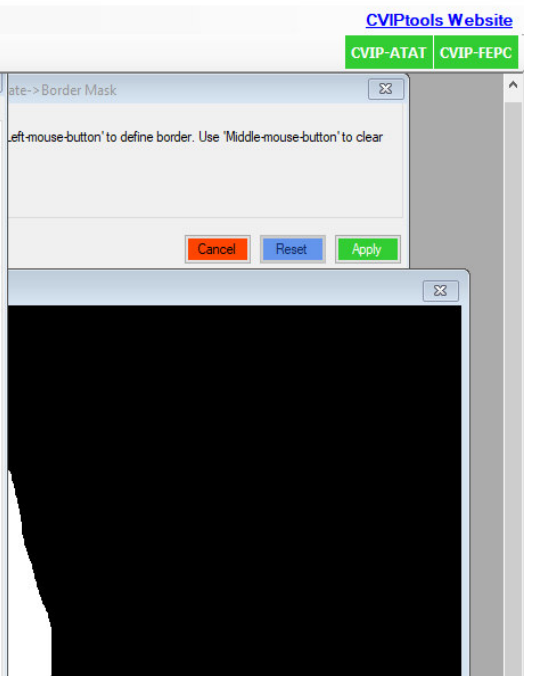

After apply nearest neighbor and K-nearest neighbor algorithm using pattern classification different rice varieties qualities features extracted. In this way different percentage of different rice quality achieved which is best for compare with each other to detect best variety quality.

\section{Result and discussion}

In this study we use three varieties of rice for detect best of quality rice. In this study we get the images of rice varieties and then these images processes in the CVIP tool which already discussed above. We give the name to all three varieties of rice as A, B, C and use nine images of each class. We get three or five border mask of each image for train dataset and two border mask for test data set. In this study we apply RST-invariant features, Histogram and texture features of different border masks of images and get sub features results which shows such as:

\section{Results of images using RST invariants Features,Histogram features and Texture Features through different border masks of images}

\begin{tabular}{|c|c|c|c|c|c|c|c|c|c|c|c|c|c|c|c|c|c|}
\hline S.No & Name & Row & Column & RST1 & RST2 & RST3 & RST4 & RST5 & RST6 & RST7 & $\begin{array}{c}\text { Histo_M } \\
\text { ean_R }\end{array}$ & $\mid \begin{array}{c}\text { Histo_M } \\
\text { ean_G }\end{array}$ & $\mid \begin{array}{c}\text { Histo_M } \\
\text { ean_B }\end{array}$ & $\mid \begin{array}{c}\text { Histo_S } \\
\text { tandard } \\
\text { Deviati } \\
\text { on_R }\end{array}$ & $\begin{array}{l}\text { Histo_S } \\
\text { tandard } \\
\text { Deviati } \\
\text { on_G }\end{array}$ & $\begin{array}{c}\text { Histo_S } \\
\text { tandard } \\
\text { Deviati } \\
\text { on_B }\end{array}$ & $\begin{array}{l}\text { Histo_S } \\
\text { kew_R }\end{array}$ \\
\hline 1 & PNG & 88 & 27 & 0.25288 & 0.03565 & 00015 & $9 \mathrm{E}-05$ & 0 & -06 & 0 & .6765 & 68.7885 & 85.709 & 14.9764 & 15.3807 & 17.2095 & 18434 \\
\hline 2 & PNG & 130 & 29 & 0.26867 & 0.04458 & 0.0001 & 3.1E-05 & 0 & $5 E-06$ & c & 5.1426 & 60.9658 & 74.6224 & $\begin{array}{l}4 \\
+14.0275 \\
\end{array}$ & 13.585 & 3.0811 & 1.91003 \\
\hline 3 & b2.PNG & 68 & 3 & 0.16884 & 0.00056 & 0.00015 & 1E-06 & 0 & 0 & 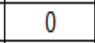 & 65.7589 & 73.0557 & 89.4266 & 20.0279 & 20.1233 & 248 & 2.82203 \\
\hline 4 & b2.PNG & 223 & 39 & 0.17135 & 0.00145 & 0.00011 & $4 \mathrm{E}-06$ & 0 & 0 & 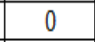 & 58.663 & 64.399 & 77.6888 & \begin{tabular}{|l|l|}
12.1544 \\
\end{tabular} & 11.7062 & .1677 & 0.90765 \\
\hline 5 & PNG & 44 & & 0.18983 & 0.008 & $2 \mathrm{E}-05$ & $5 \mathrm{E}-0$ & & 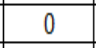 & & 80.3881 & 84.2447 & 97.4538 & \begin{tabular}{|l|l|l|}
14.8648 \\
\end{tabular} & 14.2731 & 2811 & 0.4914 \\
\hline 6 & 2.PNG & 161 & 11 & 0.18302 & 0.00596 & 0.00017 & $8 \mathrm{E}-06$ & 0 & 0 & & 84.2596 & 84.4449 & 90.6811 & 15.7965 & 15.2224 & 14.6024 & 0.68072 \\
\hline $\begin{array}{l}\text { sto_S } \\
\text { ew_G }\end{array}$ & $\begin{array}{c}\text { Histo_SS } \\
\text { kew_B }\end{array}$ & $\begin{array}{l}\text { Histo_E } \\
\text { nergy_R }\end{array}$ & $\begin{array}{c}\text { Histo_E } \\
\text { nergy_ } \\
\text { G }\end{array}$ & $\begin{array}{l}\text { Histo_E } \\
\text { nergy_B }\end{array}$ & $\begin{array}{c}\text { Histo_E } \\
\text { ntropy_ } \\
\text { R }\end{array}$ & $\begin{array}{c}\text { Histo_E } \\
\text { ntropy_ } \\
\text { G }\end{array}$ & $\begin{array}{c}\text { Histo_E } \\
\text { ntropy_ } \\
\text { B }\end{array}$ & $\begin{array}{l}\text { Texture } \\
\text { Energy } \\
\text { Average }\end{array}$ & $\begin{array}{l}\text { Texture } \\
\text { Energy } \\
\text { Range }\end{array}$ & $\begin{array}{c}\text { Inertia } \\
\text { Average }\end{array}$ & $\begin{array}{l}\text { Inertia } \\
\text { Range }\end{array}$ & $\begin{array}{c}\text { Correlat } \\
\text { ion } \\
\text { Average }\end{array}$ & $\begin{array}{l}\text { Correlat } \\
\text { ion } \\
\text { Range }\end{array}$ & $\begin{array}{c}\text { Inverse } \\
\text { Diff } \\
\text { Average }\end{array}$ & $\begin{array}{c}\text { Inverse } \\
\text { Diff } \\
\text { Range }\end{array}$ & $\begin{array}{l}\text { Texture } \\
\text { Entropy } \\
\text { Average }\end{array}$ & $\begin{array}{l}\text { Texture } \\
\text { Entropy } \\
\text { Range }\end{array}$ \\
\hline 27529 & 17993 & 0.0224 & 0.02186 & 0.01998 & 5.72211 & 5.74235 & 5.89108 & 0.00123 & .00031 & 86.6096 & 52.6675 & 0.81032 & 0.11473 & 2617 & 0.04394 & .1352 & 0.33831 \\
\hline 2.10615 & 2.37026 & 0.02403 & 0.02518 & 0.02608 & 5.63953 & 5.57826 & 5.51396 & 0.00133 & 0.00036 & 109.603 & 73.5989 & 0.70496 & 0.19399 & 0.31214 & 0.06284 & 10.0997 & 0.3658 \\
\hline 2.82189 & 2.89948 & 0.02034 & 0.02046 & 0.02005 & 5.94469 & 5.94485 & 5.98993 & 0.00132 & 0.00048 & 139.575 & 93.7199 & 0.82577 & 0.1175 & 0.35515 & 0.07704 & 10.2304 & 0.47609 \\
\hline 00992 & 0.84739 & 0.02659 & 0.02807 & 0.02825 & 5.51628 & 5.44368 & 5.41574 & 0.00199 & 0.00069 & 37.0329 & 24.2695 & 0.86519 & 0.08823 & 0.37337 & 0.07235 & 9.67364 & 0.42445 \\
\hline 32972 & 0.23541 & 0.01963 & 0.02023 & 0.02035 & 5.88817 & 5.8499 & 5.85685 & 0.00116 & 0.00037 & 58.9245 & 46.6674 & 0.85229 & 0.11924 & \begin{tabular}{|l|l} 
& 0.33814 \\
\end{tabular} & 0.06896 & 10.3131 & 0.45275 \\
\hline 59334 & 0.61053 & 0.01908 & 0.01953 & 0.02075 & 5.96729 & 5.9245 & 5.8523 & 0.00098 & 0.00034 & 68.8614 & 55.2415 & 0.85125 & 0.11956 & 0.31615 & 0.07181 & 10.5607 & 0.48569 \\
\hline
\end{tabular}

\section{RST-invariant features, Histogram features and Texture features}

RST Invariant, Histogram and Texture features and get the results using different sub features of following three main features. In this way we get result of every image using different borders of image. After getting the train data sets and test data sets we use pattern classification and apply nearest neighbor and K-nearest neighbor algorithm for final results. 


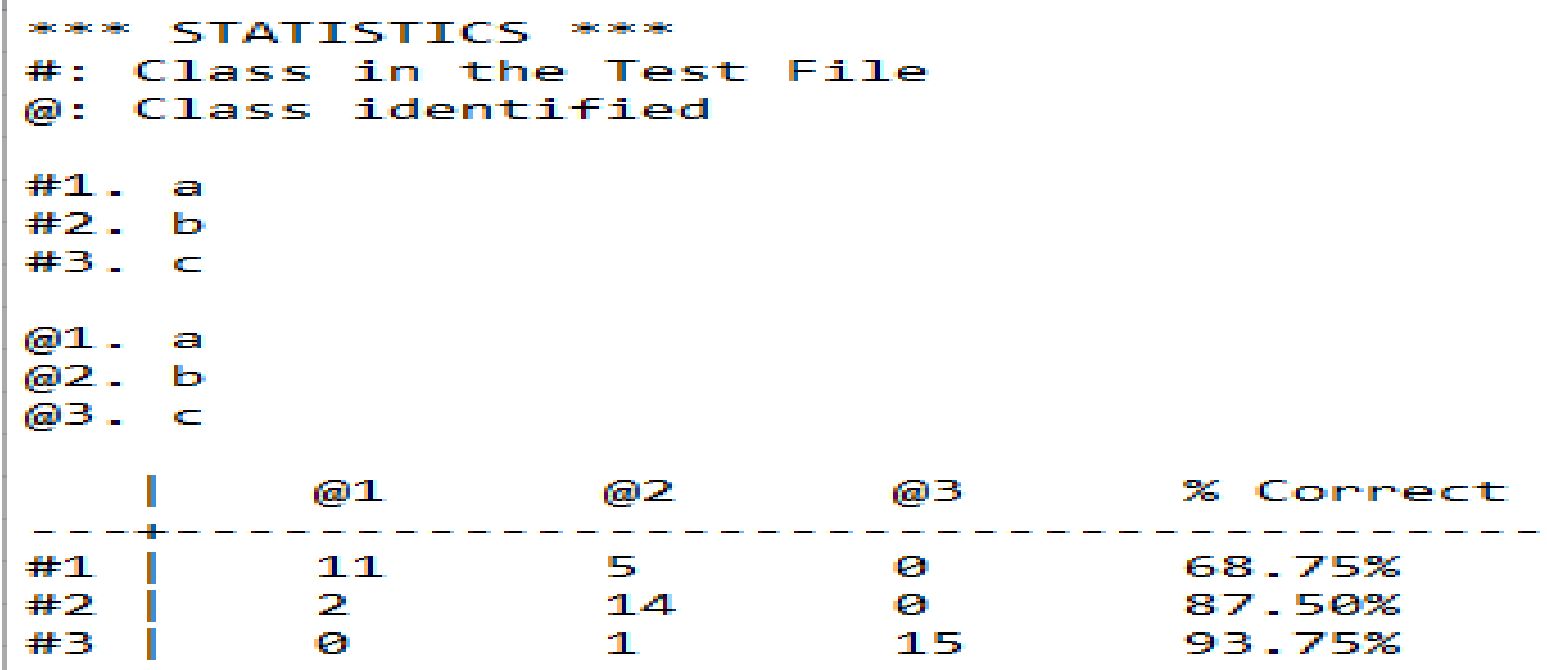

Nearest neighbor Pattern Classification results

in above diagram nearest neighbor pattern classification results show the rice c kind is best which show more than percentage other $a$ and $b$.

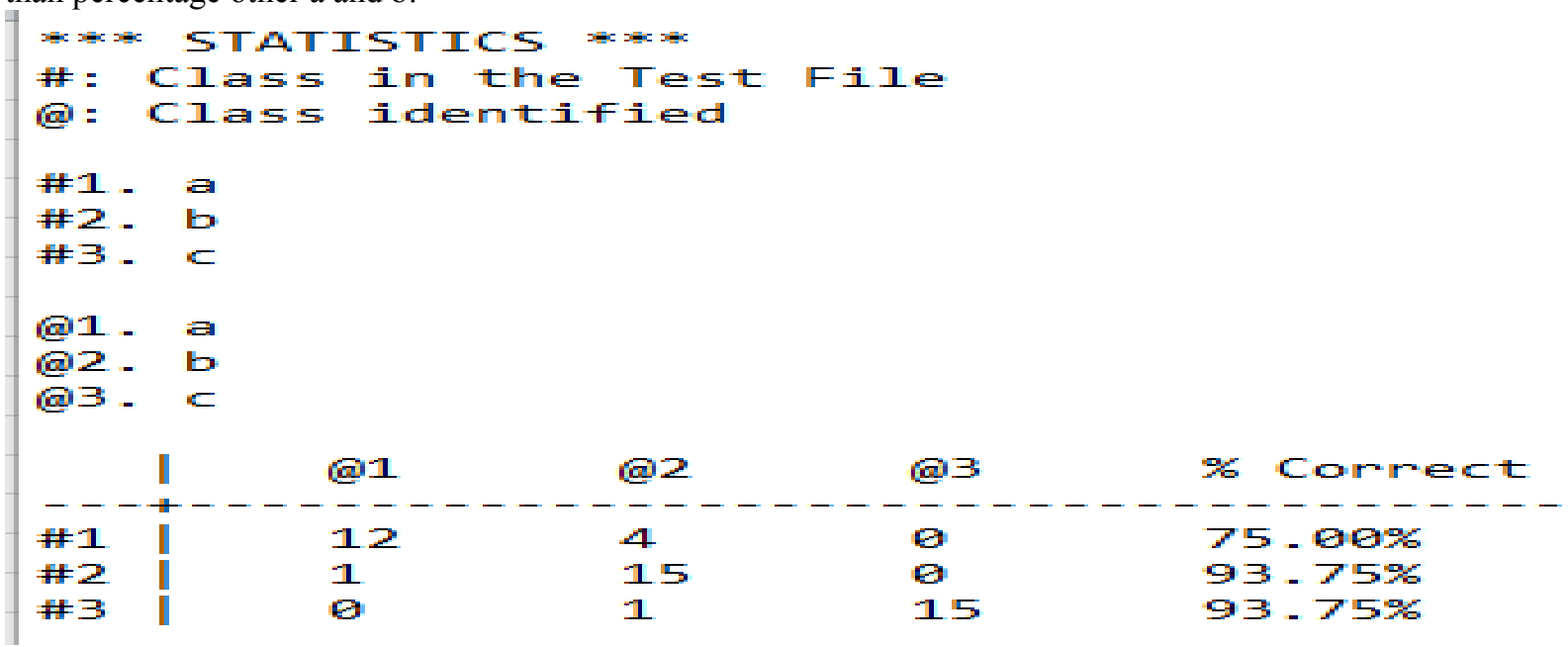

K-Nearest Neighbor Pattern classification results using $K=2$

It's clear in above if we chose pattern classification through $\mathrm{K}$-nearest neighbor algorithm using $\mathrm{K}=2$ then $\mathrm{b}$ and $\mathrm{c}$ kind of rice best result.

In above chart the final pattern classification results will be shown as percentage wise. These results are the best for classification.

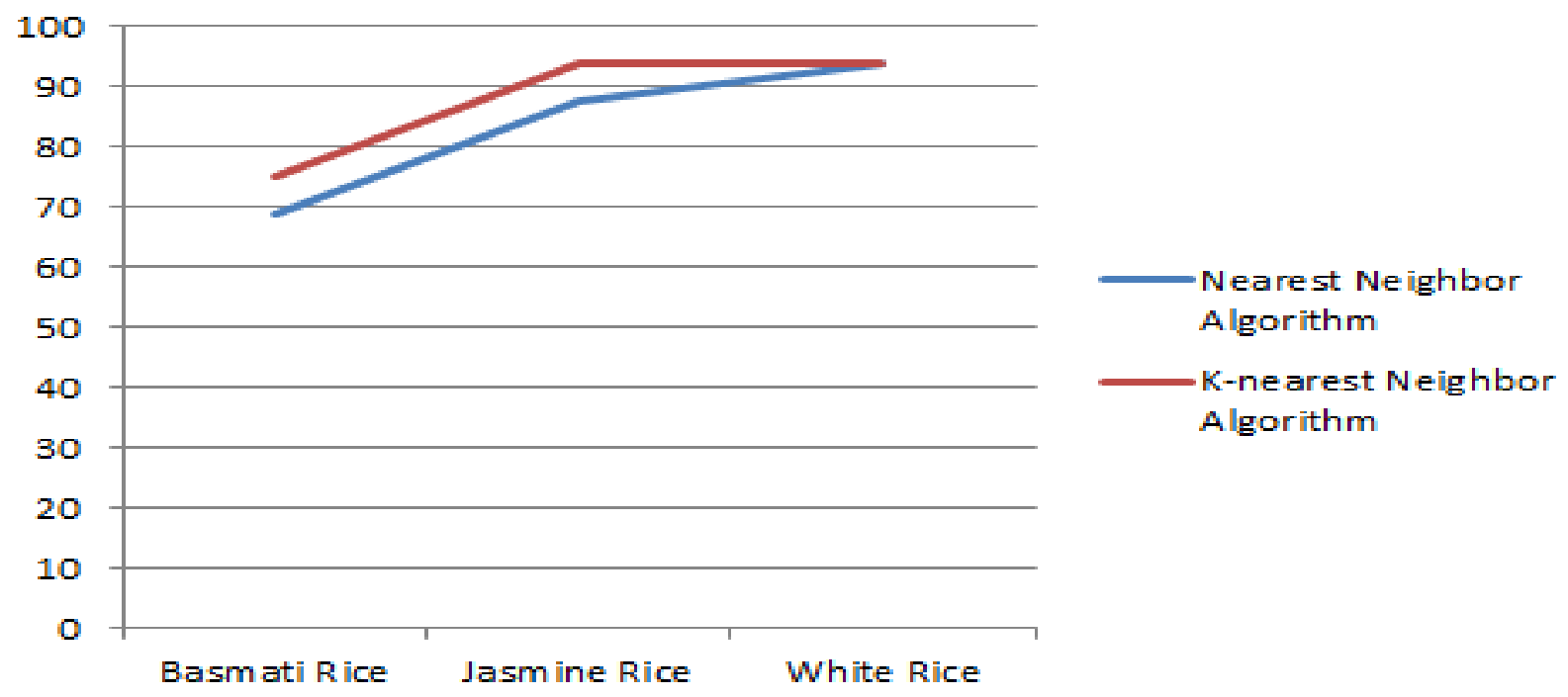


In this study three varieties of rice were discriminated on the basis of quantitative parameters will be taken. The RST-Invariant, Histogram and texture features were implemented for the analysis of images which made our approach faster than the other approaches in which morphological, Color and other textural features are used. Knearest neighbor (KNN) and nearest neighbor methods were implemented very successfully for the discrimination of three varieties of rice. When we use distance one of train and test the final result percentage of nearest neighbor are shown in fig $\mathrm{A}=68.75 \%, \mathrm{~B}=87.50 \%, \mathrm{C}=93.75$. in this way when we apply $\mathrm{K}$-nearest neighbor algorithm the result shown $\mathrm{A}=75.00 \%, \mathrm{~B}=93.75 \%, \mathrm{C}=93.75 \%$. After apply both algorithm the variety $\mathrm{C}$ (White rice) result is $93.75 \%$ which is the best pattern classification result. In this study we predict the White rice quality is better than Basmati and Jasmine rice. This result of k-nearest neighbor is also same as nearest neighbor.

\section{Future work}

The RST-Invariant, Histogram and texture features were implemented for the analysis of images which made our approach faster than the other approaches in which morphological, Color and other textural features are used. Knearest neighbor (KNN) and nearest neighbor methods were implemented very successfully for the discrimination of three varieties of rice. The goal was to develop algorithms to detect the different rice quality verities. The second goal is to propose a new domain among the different varieties of rice on quality base. In this study CVIP tool is used to obtain the result by implementing the different algorithms other tools are also available like MATLAB, Mazda etc for future work for best analysis.

\section{References}

[1] Ricepedia, "Rice around the world - Ricepedia," Ricepedia.

[2] wikipeadia, "Rice production in Pakistan - Wikipedia.".

[3] T. Avudaiappan, S. Sangamithra, A. Silpha, S. Sherin, and K. M. Visalakshi, “Analysing Rice Seed Quality Using Machine Learning Algorithms," pp. 22-26, 2019.

[4] C. Sewwandi Silva and U. Sonnadara, "Classification of Rice Grains Using Neural Networks," Proc. Tech. Sess., vol. 29, no. March 2013, pp. 9-14, 2013.

[5] V. Becerra, M. Paredes, E. Gutiérrez, and C. Rojo, "Genetic diversity, identification, and certification of Chilean rice varieties using molecular markers," Chil. J. Agric. Res., vol. 75, no. 3, pp. 267-274, 2015.

[6] P. Dheer and R. K. Singh, "Identification of Indian rice varieties using machine learning classifiers," Plant Arch., vol. 19, no. 1, pp. 155-158, 2019.

[7] M. Tariq, M. Ibrahim, N. Ahmad, and A. M. Ahmad, "Identification of Canola Seeds using Nearest Neighbor and K-Nearest Neighbor Algorithms,” vol. 6, no. 10, pp. 36-42, 2015.

[8] H. Zareiforoush, S. Minaei, M. R. Alizadeh, and A. Banakar, "Qualitative classification of milled rice grains using computer vision and metaheuristic techniques," J. Food Sci. Technol., vol. 53, no. 1, pp. $118-131,2016$

[9] Z. Ye, L. Ma, and H. Chen, "A hybrid rice optimization algorithm," ICCSE 2016 - 11th Int. Conf. Comput. Sci. Educ., no. Iccse, pp. 169-174, 2016.

[10] R. Birla and A. P. S. Chauhan, "An Efficient Method for Quality Analysis of Rice Using Machine Vision System," J. Adv. Inf. Technol., vol. 6, no. 3, pp. 140-145, 2015.

[11] 105-110. Bora G C, Pathak R, Ahmadi M, Mistry P. 2018. Image processing analysis to track colour changes on apple and correlate to moisture content in drying stages. Food Quality and Safety 2, "Classification of Milled Rice Using Image Processing," vol. 8, no. 2, pp. 10-14, 2017.

[12] M. Senthil Kumar and M. Javeed, “An efficient rice variety identification scheme using shape, harlick \& color feature extraction and multiclass SVM," Int. J. Eng. Adv. Technol., vol. 8, no. 6, pp. 3629-3632, 2019.

[13] D. I. Patrício and R. Rieder, "Computer vision and artificial intelligence in precision agriculture for grain crops: A systematic review," Comput. Electron. Agric., vol. 153, no. August, pp. 69-81, 2018.

[14] A. Ganatra and A. Jadhav, "Rice QA using Deep Learning," no. May, pp. 5370-5372, 2019.

[15] V. K. Shrivastava, M. K. Pradhan, S. Minz, and M. P. Thakur, "Rice plant disease classification using transfer learning of deep convolution neural network," Int. Arch. Photogramm. Remote Sens. Spat. Inf. Sci. - ISPRS Arch., vol. 42, no. 3/W6, pp. 631-635, 2019.

[16] R. H. Ajaz, L. Hussain, A. Jammu, and K. Muzaffarabad, "Seed Classification using Machine Learning Techniques," J. Multidiscip. Eng. Sci. Technol., vol. 2, no. 5, pp. 3159-40, 2015.

[17] N. Nagoda and L. Ranathunga, "Rice Sample Segmentation and Classification Using Image Processing and Support Vector Machine," 2018 13th Int. Conf. Ind. Inf. Syst. ICIIS 2018 - Proc., no. 978, pp. 179$184,2018$.

[18] M. Tariq and M. Ibrahim, "Bark Classification of Trees Using K-Nearest Neighbor \& Nearest Neighbor Algorithms," Comput. Eng. Intell. Syst. , vol. 7, no. 1, pp. 32-42, 2016. 
[19] M. Hasham, "Identification of Canola Seeds through Computer Vision Image Processing," vol. 7, no. 10, pp. 1-7, 2017.

[20] P. Dheer, "Distinguishing of Rice Varieties by Using Machine Learning Models," Ijarcce, vol. 8, no. 1, pp. 55-57, 2019. 